\title{
Acerca da métrica da percepção do espaço visual
}

\author{
On the metric of visual space
}

\author{
José Aparecido Da Silva ${ }^{1}$ \\ José Antonio Aznar-Casanova ${ }^{2}$ \\ Nilton Pinto-Ribeiro Filho ${ }^{3}$ \\ Javier Enrique Santillán ${ }^{4}$
}

${ }^{1}$ Departamento de Psicologia e Educação da Universidade de São Paulo - USP - Ribeirão Preto (SP) - Brasil.

${ }^{2}$ Departamento de Psicologia Básica Facultad de Psicologia, Universidad de Barcelona - España.

${ }^{3}$ Instituto de Psicologia da Universidade Federal do Rio de Janeiro - URFJ - Rio de Janeiro (RJ) - Brasil.

${ }^{4}$ Departamento de Luminotecnia, Luz y Visión Facultad de Ciencias Exactas y Tecnología da Universidad Nacional de Tucumán - Argentina.

Endereço para correspondência: José Aparecido da Silva - Departamento de Psicologia e Educação - Av. Bandeirantes 3900 - Campus da USP - Ribeirão Preto (SP) CEP 14040-901

E-mail: jadsilva@ffclrp.usp.br

Recebido para publicação em 03.10.2005

Versão revisada recebida em 18.11.2005

Aprovação em 22.11.2005

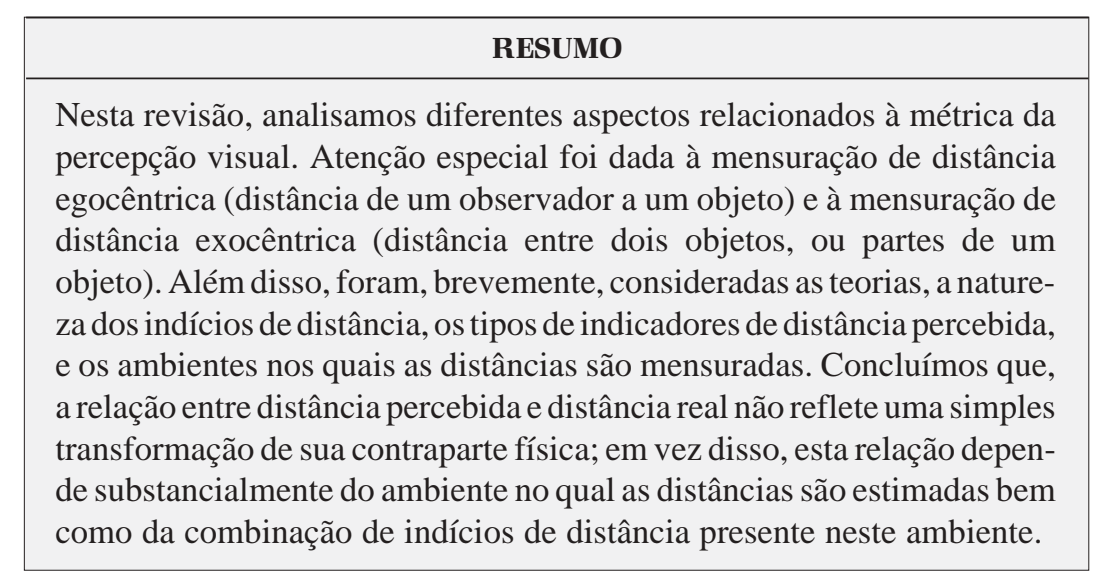

Descritores: Percepção de profundidade; Percepção espacial; Percepção visual; Percepção de distância; Psicofísica; Ilusões ópticas

\section{INTRODUÇ̃̃̃O}

O entendimento acerca da percepção de distância tem se constituído um dos problemas fundamentais no estudo da percepção visual. Apesar de uma longa história de investigação, a percepção de distância ainda não está completamente elucidada e continua sendo uma área de pesquisa ativa, com amplas aplicações na medicina, na ergonomia, no treinamento militar e nas pesquisas espaciais em ambientes reais e virtuais, etc.

Para investigar a percepção de distância, temos que considerar os mecanismos que estão subjacentes a ela. Como os humanos podem interagir com ambientes que eles "vêem" mesmo com os seus olhos fechados, nós podemos assumir que eles têm uma representação interna do espaço ao redor deles. Tradicionalmente, esta representação interna tem sido denominada de espaço visual, e várias pesquisas têm investigado suas propriedades ${ }^{(1)}$.

Nesta revisão da literatura acerca da percepção visual do espaço, propomo-nos, de um lado, considerar o valor da eficácia relativa (à tarefa e à distância) de um conjunto de indícios de distância e profundidade e verificar o modelo de estrutura de espaço visual em vários subespaços (ambientes nos quais as distâncias são estimadas; distâncias pequenas e grandes), nos quais cada indício mostra um valor de utilidade relativa diferenciado. Por outro lado, verificar se dispomos de uma geometria intrínseca própria do sistema visual ou, pelo contrário, se existe um relativismo geométrico adaptativo.

\section{Da teoria}

Tem havido também vários enfoques para investigar a estrutura do espaço visual. J. J. Gibson acreditava que a percepção visual era verídica e direta, implicando que não é necessário um espaço visual, haja vista que 
tudo que é visível num ambiente natural, estruturado, é direta e imediatamente disponível ${ }^{(2)}$. Gilinsky também investigou a relação entre o mundo real e a nossa representação dele, procurando equações matemáticas que mapeassem o espaço físico ao espaço visual ${ }^{(3)}$. Recentemente, Indow realizou uma análise intensa e profunda sobre a estrutura geométrica e global do espaço visual ${ }^{(4)}$.

Loomis et al., ao longo dos últimos 15 anos, realizaram inúmeros experimentos envolvendo ação visualmente dirigida, na qual aos observadores é dado um mesmo padrão visual e, em seguida, são solicitados a desempenhar uma tarefa sem visão. Uma teoria proposta por Loomis et al., sugere que o espaço físico é mapeado em espaço visual, gerando medidas muito acuradas de distância egocêntrica, mas não de distância exocêntrica. Loomis et al., usaram este padrão de resultados para argüirem que a percepção visual e a ação visualmente dirigida delineiam-se sobre os mesmos indícios perceptuais ${ }^{(1)}$.

Uma outra teoria, que inicialmente parece contradizer a concepção de um sistema unificado entre a percepção visual e a percepção visuo-motora, proposta por Loomis et al., é aquela proposta por Goodale, Milner, os quais mostraram várias evidências neurológicas e comportamentais para uma separação entre as vias neurais conduzindo à percepção visual consciente, e aquelas levando à ação visualmente dirigida ${ }^{(5-6)}$. Esta teoria compete com aquela de Loomis et al., porque sua sugestão de separar as vias visuais e visuo-motoras implica alguma dissociação do espaço visual em dois. Michaels ,um psicólogo ecologista, ataca as definições de percepção e ação perceptual de Goodale e Milner, apontando que a suposição de um fluxo de processamento de informação puramente perceptual vai contra o hermético acoplamento entre percepção-ação advogado pelos psicólogos baseados na tradição ecológica ${ }^{(7)}$. Apesar desta objeção, a maioria dos pesquisadores em percepção acha a suposição de Goodale, Milner irrepreensível ${ }^{(5)}$.

Ainda que estas duas teorias pareçam ser conflitantes, nenhuma exclui, de fato, a outra. Goodale e Milner notam que enquanto as duas vias podem operar independentemente, há definitivamente alguma comunicação entre elas ${ }^{(5)}$. Por conseguinte, isto seguiria a simples formulação de Loomis et al., de que a representação egocêntrica do espaço visual reside em um lugar, e pode estar disponível a ambas as vias ${ }^{(1)}$.

\section{Da mensuração de distância percebida}

Sabemos, a partir das investigações psicofísicas, que nossa percepção do espaço não é verídica ${ }^{(8-9)}$. Concretamente, parece que o espaço percebido (espaço visual) torna-se mais comprimido conforme aumenta-se a distância de observação ${ }^{(10-11)}$. Além disso, também se tem constatado que os diferentes procedimentos utilizados para julgar distância produzem algumas variações nos resultados destes experimentos. E um bom indicador do grau de compressão do espaço é o expoente da função de potência ${ }^{(12)}$. Isto é: $\mathrm{D}^{\prime}=\mathrm{k}^{*} \mathrm{D}^{\mathrm{n}}$. Onde $\mathrm{D}^{\prime}$ é a distância percebida, $\mathrm{D}$ é a distância física ou real, $\mathrm{k}$ é uma constante (relacionada com a escala ou unidades de medida utilizadas nas estimativas) e o expoente $\mathrm{n}$ é um indicador da finura discriminativa do observador em função da magnitude da intensidade do estímulo (distância). Em outras palavras, o expoente informa sobre a amplitude de valores nos quais o ajuste do sensor à realidade física se acopla (correspondência) com maior ou menor precisão, refletindo, pois, a taxa de compressão da distância percebida em relação à distância física. Portanto, o valor de $\mathrm{n}$ é um parâmetro importante que reflete a aceleração da função relacionando estimativas de distância às distâncias físicas, e indica o grau de constância de distância. Distância percebida é uma função linear da distância física quando $n$ é igual a 1,0, e neste caso é dito ocorrer uma constância de distância perfeita. De outro lado, se n > 1,0, há uma aceleração positiva na função que indica superconstância, e se $\mathrm{n}<1,0$, há uma aceleração negativa que indica subconstância.

Tradicionalmente, os pesquisadores têm assumido que a distância percebida é direta e linearmente relacionada à distância física, isto é, com $\mathrm{n} \approx 1$, e que, supostamente, há uma perfeita constância de distância. Mas, sem dúvida, isto não corresponde aos dados psicofísicos disponíveis ${ }^{(13)}$. Certamente, o valor do expoente é próximo da unidade quando o objeto está próximo do observador, mas também se tem constatado que este valor diminui com a distância. Assim, hoje dispomos de dados que mostram que, para diferentes amplitudes de distâncias, o expoente varia entre 1 e 0,65 (para uma amplitude de até $300 \mathrm{~m}$ em relação ao observador) e pode alcançar 0,36-0,40 (para distâncias um pouco acima de $2 \mathrm{~km}$ ). Nesta amplitude com grandes distâncias, Flückiger solicitou a seus observadores que julgassem a distância na qual estavam barcos flutuando no Lago Lèman (observando-os a partir de Genebra em direção a Montreux) numa amplitude entre $200 \mathrm{me}$ $2,25 \mathrm{~km}^{(14)}$. Os únicos indícios visuais disponíveis foram o tamanho relativo e a altura sobre o campo visual (elevação); ainda que, talvez, tenha havido a perspectiva aérea e a densidade de textura sobre as ondas do mar. Os dados mostraram que o valor do expoente "n", médio, para distâncias em torno de $2 \mathrm{~km}$ foi de $0,38^{(14)}$.

Entretanto, dentro do domínio da percepção do espaço, um dos mais salientes aspectos enfocado por Loomis, et al., é a diferença entre percepção de distância egocêntrica e exocêntrica. A distância egocêntrica é a distância do observador até alguma localização no mundo, enquanto distância exocêntrica é a distância entre dois objetos. Eles argumentam que o espaço visual é organizado em termos de distância egocêntrica, e mostram evidências para a quase perfeita acurácia da percepção de distância egocêntrica, e significativamente uma menor acurácia para a percepção de distância exocêntrica ${ }^{(1)}$.

Outro aspecto que tem preocupado Da Silva, que, embora controlado, não tem sido discutido com detalhes ${ }^{(13)}$, é o indicador de distância percebida. Distância pode ser indicada pelo observador implicitamente ou explicitamente. A indicação implícita de distância percebida envolve uma ação, tal como caminhar em direção a um objeto ou atirar um alvo à distância de um objeto (ou objetos), a qual indica indiretamente a distância que o observador acredita estar percebendo. A indica- 
ção explícita de distância percebida tende a ser mais cognitiva, e envolve um registro direto da distância que o observador acredita estar percebendo (ver, por exemplo, os trabalhos de Gogel, Da Silva) ${ }^{(15-16)}$.

\section{Da modelagem-ajustamento dos dados}

Um outro aspecto importante que tem chamado a atenção de Loomis et al., é como modelar os dados após eles terem sido coletados. Um método amplamente empregado de modelagem de dados obtidos acerca da percepção de distância é a função de potência. $\mathrm{O}$ uso de uma função de potência geralmente focaliza-se sobre o expoente o qual indica a taxa em que a distância percebida parece aumentar em relação ao aumento da distância física, real. Os estudos realizados por Teghtsoonian e Teghtsoonian ilustram este método de modelagem de dados de distância percebida ${ }^{(17-18)}$. No primeiro destes estudos, julgamentos verbais de distâncias em ambiente natural fechado foram dados pelos observadores, e suas respostas foram modeladas como uma função de potência. No segundo estudo, o expoente médio encontrado no estudo original foi comparado com o expoente médio obtido em ambiente natural aberto. No estudo em ambiente fechado o expoente médio foi de 1,2, indicando que distância percebida aumenta mais rapidamente do que a distância real demarcada em laboratório. No estudo envolvendo espaço aberto, os expoentes médios ficaram entre 0,85 e 0,99 , diminuindo quando a amplitude de distâncias julgada aumenta, o que significa que distância percebida e distância real aumentam numa mesma taxa até um dado ponto, após o qual o aumento na distância real tem um impacto decrescente sobre a distância percebida. Há exatamente vinte anos, Weist e Bell ${ }^{(19)}$ Da Silva revisou inúmeros estudos que utilizaram a função de potência como forma de modelagem de dados de distância percebida, indicando a sua importância, utilidade e popularidade ${ }^{(13)}$. Todavia, a popularidade da função de potência para modelar dados de distância tem declinado nos últimos anos, em favor de modelos lineares, mais simples, relacionando distância percebida e distância física, real, tal como usada por Loomis et $\mathrm{al}^{(1)}$. Modelos lineares modelam mais diretamente a percepção de distância absoluta, enquanto modelos de função de potência são mais úteis para entender como a percepção de distância muda em função da distância física. Em outras palavras, os modelos lineares mostram mais diretamente o mapeamento (ajustamento) entre o real e o percebido, ao passo que a função de potência mostra uma mudança em termos da taxa de aceleração da função relacionando distância percebida á distância real ou física.

\section{Dos indícios de distância}

Um outro aspecto que merece consideração trata-se de quais são exatamente os indícios de profundidade que estão disponíveis para o sistema visual humano.

Há duas espécies de informação que os indícios de distância podem fornecer. A maioria dos indícios fornece informação de distância relativa, exocêntrica (intervalo entre objetos ou partes de um mesmo objeto), ou ordinal, informando ao siste- ma visual sobre as relações de distância entre diferentes objetos no mundo. Por exemplo, um indício de distância relativa pode informar ao sistema visual que um objeto está duas vezes mais distante do que um outro objeto, mas não exatamente o quão distante cada objeto está. Poucos indícios fornecem informação de distância absoluta, indicando ao sistema visual a quantidade exata de distância no mundo. Estes indícios de distância absoluta são freqüentemente limitados, ou pela eficácia de sua amplitude, ou pela familiaridade com o objeto observado. Os modos com os quais os indícios absolutos (egocêntricos) e relativos (excêntricos) de distância (em ambientes em que o observador permanece numa posição fixa em relação ao que está sendo observado), para a percepção de distância podem ser combinados têm sido analisados por Cutting e Vishton ${ }^{(20)}$. Dentre os indícios analisados está a acomodação, a qual fornece informação útil para objetos dentro de aproximadamente 2 a 3 metros do observador ${ }^{(21)}$; pois, em distâncias maiores, este indício já se encontra em seu ponto máximo e nada mais contribui para a localização exata da distância percebida egocêntrica ou exocêntrica. Na verdade, a quantidade com a qual estes indícios influenciam a percepção varia com a distância.

Para a percepção do espaço, Cutting e Vishton ${ }^{(20)}$ entenderam que é útil dividir o ambiente ao redor do observador em classificações de distância: espaço peripessoal ou manipulatório (dentro do alcance dos braços), espaço de ação (2 a 30 m) e espaço em perspectiva (vista espaço- maior que 30 metros). Dentro do espaço de ação, a acomodação, a convergência, e o movimento paralaxe são considerados indícios fracos para distância egocêntrica ${ }^{(22-23)}$, pois, seus efeitos individuais tendem a diminuir além de dois metros. Todavia, distância ou profundidade egocêntrica, além de dois metros, podem ser recuperadas pela disparidade binocular usando a convergência como um fator escalar ${ }^{(24)}$. Todavia, parece que a eficácia da convergência dos dois olhos como um indício de profundidade para distância egocêntrica diminui drasticamente se a distância é maior que seis metros. Há, também, evidências de que para distâncias pequenas, os indícios de distâncias na superfície do terreno (gradientes de textura) são importantes para perceber distâncias muito grandes ${ }^{(25)}$.

Outros indícios de distância são dados pelo movimento. Um de tais indícios, o movimento paralaxe, permite ao observador determinar as distâncias relativas de dois objetos baseados em seu movimento relativo quando o observador movese de sua posição de observação através do mundo. Objetos que estão próximos ao observador parecerão mover-se mais que objetos distantes do observador. Outro indício de movimento deriva-se do movimento de textura ou de fluxo óptico, quando o observador se move no mundo. Este indício é dado pela maneira com que a textura muda em função do tempo, ou quando o observador, ou o mundo, se move. Quando as texturas se comprimem, cobrem menos área e aumentam em densidade, os objetos associados a elas distanciam-se mais. $\mathrm{O}$ gradiente de textura também pode ser um indício de profundidade sem movimento. Desde que a densidade de textura de um 
objeto tende a ser muito maior em partes do objeto que estão mais distantes, o gradiente de textura pode funcionar como um indício de distância relativa.

Oclusão ou sobreposição é também um indício relevante que opera numa grande amplitude de distâncias. Provavelmente, este é o único indício capaz de determinar um ordenamento no espaço visual em grande escala. Todavia, este indício fornece-nos apenas informação ordinal.

Uma outra propriedade da projeção de um espaço tridimensional a um espaço bidimensional é a existência de uma linha no horizonte, no nível do olho, infinitamente distante, em direção da qual todas as linhas paralelas convergem. A trigonometria básica permite ao sistema visual determinar a distância do observador a um objeto num terreno plano, à medida que o ângulo entre o objeto e o horizonte, e a altura do observador, sejam conhecidas ${ }^{(26)}$. Todavia, em relação à linha no horizonte é necessário diferenciar entre objetos sobre a linha do horizonte e objetos flutuando sobre a linha do horizonte, haja vista que as regras mudam em ambos os casos. No primeiro caso (objetos na linha do horizonte como, por exemplo, pessoas), quanto mais próxima à linha do horizonte estiver uma pessoa mais distante ela será percebida. Todavia, no segundo caso (objetos flutuando acima da linha do horizonte como, por exemplo, nuvens), ao contrário, quanto mais distante estiver a linha do horizonte tanto mais próxima é a nuvem percebida.

Ooi et al., recentemente demonstraram que a declinação angular a partir da linha horizontal a um objeto é um indício de distância ${ }^{(26)}$. Os objetos parecem próximos a um observador quando o ângulo entre o objeto e o horizonte é maior. Isto ocorre porque a linha do horizonte é geralmente constante, como é o ângulo observador entre o horizonte e os pés do observador. Como resultado, quando o ângulo entre o horizonte e um objeto aumenta, isto implicitamente diminui o ângulo entre o objeto e os pés do observador, implicando que o objeto está mais próximo.

A altura dos olhos também pode funcionar como um indício de distância. A distância pode ser determinada se tanto a altura observada quanto a altura real são conhecidas. Um indício muito simples é baseado nos tamanhos observados dos objetos. Este indício, tamanho relativo, é o entendimento do sistema visual de que objetos maiores tendem a estar mais próximo ao observador do que objetos menores. Um outro indício de distância, o tamanho familiar, funciona para objetos de tamanho conhecido. Se um objeto cujo tamanho é familiar ao observador for visto em alguma distância, o observador usa a diferença entre o tamanho conhecido do objeto e seu tamanho percebido para apropriadamente escalar a distância percebida em distância real. Tamanho familiar é o único indício para distância que fornece estimativas de distância egocêntrica (absoluta), mais do que relativa, sem necessidade de repousar em sinais motores dos músculos oculares. Gogel, Da Sil$\mathrm{va}^{(15-16)}$ elaboraram uma teoria que, usando o tamanho familiar para estimativas de distância egocêntrica e de tamanho, dis- tingue os fatores cognitivos dos tipicamente perceptuais que afetam os indicadores de respostas espaciais. Esta teoria tem sido comprovada mesmo quando julgamentos de intervalos exocêntricos são efetuados em ambiente de campo aberto, comparando-se os julgamentos de tamanho de objetos familiares e não-familiares apresentados em condições naturais e em grandes distâncias físicas ${ }^{(27-28)}$.

Enquanto muitos indícios de distância são bem estabelecidos, há muitos outros indícios que não têm sido tomados em consideração. Ao lado disso, muitos indícios combinam-se e muitos deles são ponderados de forma diferente, em diferentes ambientes, real, virtual e pictórico, e atuam de forma diferente à medida que a distância real aumenta. Por isso, é importante também, analisar o ambiente visual em que a percepção de distância ocorre.

\section{Da mensuração de distância percebida em ambiente real}

Numerosos tipos de julgamentos têm sido usados ao longo dos anos ${ }^{(13,21,29)}$ para indicar percepção de distância, mas recentemente muita atenção tem sido dada ao uso de tarefas envolvendo ação visualmente dirigida. Caminhar sem-visão, isto é, caminhar com a visão-oclusa, em direção a um objeto é um exemplo típico deste tipo de comportamento. Numa tarefa típica de caminhar em direção ao alvo, o observador vê um alvo colocado no ambiente em alguma distância dele (previamente vista). Quando pronto, ele é instruído para colocar uma venda nos olhos, imediatamente fechar seus olhos, e, em seguida, caminhar a um alvo previamente observado. Este procedimento de estimação é denominado de ação visualmente dirigida ${ }^{(1)}$. A distância caminhada é usada como um indicador da distância inicialmente percebida do alvo e reflete sua percepção da localização do alvo. Esta distância caminhada é comparada com a distância real para determinar a acurácia global do desempenho ${ }^{(30)}$.

Em ambientes com indícios completos de distância (ambiente em que todos os indícios ou combinações de indícios estão usualmente presentes), os resultados dos experimentos usando a tarefa de caminhar visualmente dirigida demonstram que, em média, os humanos são muito bons em desempenhar esta tarefa ${ }^{(1,31-35)}$. Por exemplo, Loomis et al., encontraram desempenho acurado quando solicitaram aos observadores para caminharem sem-visão a diferentes alvos colocados a, até, 12 metros distantes ${ }^{(1)}$. Baseados em tais achados, teorias têm emergido considerando quão hábeis são as pessoas para manter a consciência de sua orientação e direção enquanto caminhando sem-visão. Rieser tem sugerido que há um estreito acoplamento entre a representação visual do observador do ambiente e suas interações nele ${ }^{(35)}$. Esta representação visual pode ser "atualizada" pelo observador - mesmo na ausência de visão - e possibilita navegação acurada dentro do espaço. Assim, as distâncias caminhadas pelos observadores em tarefas de caminhar sem-visão também refletem a habilidade para atualizar imagens internalizadas do ambiente, especialmente com respeito ao alvo. 


\section{Da mensuração de distância percebida em ambiente em grande escala}

Relacionada à percepção de distância egocêntrica é a percepção em grande escala. A diferença está na ênfase, com distância egocêntrica sendo associada a um único e simples alvo, e distância em grande escala sendo associada com uma cena ou cenário inteiro. A questão central resume-se em entender no que se baseia o observador para perceber a diferença entre uma cena visual complexa e cópias da cena variando apenas em termos de escala como, por exemplo, configurando uma cena num espaço pictórico-fotográfico, num espaço virtual, ou em pequenas escalas construídas em aléias visuais em laboratórios, ou mesmo usando representações cognitivas de grandes espaços, como mapas ${ }^{(20,36-37)}$. Em particular, perguntamos que tipo de processamento perceptual e cognitivo está envolvido quando uma pessoa constata a imensidão no ambiente visível. Por exemplo, quando observamos um grande objeto numa cena natural, tais como a Torre Eiffel em Paris, a Ponte Rio-Niterói e o Estádio Maracanã no Rio de Janeiro, nós usualmente nos impressionamos com a enormidade de tais objetos. Mesmo a grande formação de nuvens, vista de um avião, onde os indícios binoculares e a informação de movimento paralaxe são menos úteis para especificarem a escala ou fornecerem informações de distância egocêntrica, pode parecer imensa. Embora seja muito provável que percebemos estes enormes objetos bem menores do que realmente eles são, nós ainda comumente percebemos a imensidão no mundo natural.

Embora a experiência de imensidão seja comum, ela tem sido negligenciada pelos perceptólogos, talvez, porque nós, simplesmente, atribuímos imensidade aos próprios objetos. Todavia, devemos investigar e analisar qual informação do estímulo sustenta a experiência de imensidade. Quais indícios de distância são ponderados pelos observadores e qual modelo perceptual melhor explica a percepção de imensidão? Talvez, a mensuração de distâncias egocêntricas e exocêntricas em ambientes virtuais que espelham de maneira mais realística possível os ambientes real, natural, possa constituir-se numa importante ferramenta para entender qual informação o estímulo carrega que provoca nossas impressões de imensidão, de imensidade.

\section{Da mensuração de distância percebida em ambiente virtual}

Nos últimos anos, ambientes virtuais têm sido introduzidos nos experimentos de percepção de distância como um outro meio de estudar percepção do espaço. Ambientes virtuais são úteis por várias razões. Eles permitem manipulações experimentais que podem ser difíceis ou impossíveis de ser implementadas no mundo real. Eles também podem revelar mecanismos da percepção do mundo real pela comparação com a percepção em mundos virtuais. No global, os benefícios dos dispositivos de realidade virtual em pesquisa têm se tornado bastante comuns no estudo da percepção espacial ${ }^{(38-39)}$. Há vários tipos de dispositivos de realidade virtual disponí- veis para estudar percepção do espaço. Um dos mais comuns utiliza um aparelho montado sobre a cabeça (Head-MountedDisplay - HMD) - similar a um capacete com duas telas colocadas em frente dos olhos para observar imagens virtuais. Acompanham o HMD um computador e um sensor de posição atrás da cabeça. Muitos HMDs fornecem imagens estereoscópicas que ajudam a criar um cenário mais realístico possível de profundidade em ambientes virtuais, os quais têm sido útil para investigar várias dimensões espaciais como distância e $\operatorname{tamanho}^{(40)}$.

Tarefas de caminhar em direção ao alvo têm sido usadas como indicadores de percepção de distância usando HMDs. Todavia, diferentemente dos experimentos de percepção de distância em ambiente real, os resultados destes estudos mostram uma substancial compressão na distância indicada. O desempenho global tem variado amplamente entre os estudos, mas todos refletem subestimação de distância. Esta compressão sistemática em HMDs tem levado a diferentes explicações para as diferenças de desempenho entre os ambientes real e virtual. A compressão de distância na realidade virtual é um fenômeno confuso que tem resistido à investigação. Nenhuma variável tem sido encontrada que afeta a magnitude da compressão de distância, nem tem qualquer aspecto da realidade virtual sido encontrado que induz a compressão de distância em ambientes reais. Entre as explicações propostas está o efeito limitado dos campos visuais, resoluções espaciais limitadas dos dispositivos virtuais, textura limitada ou qualidade gráfica, incapacidade do observador ver o seu próprio corpo no dispositivo virtual e outros fatores levando à falta de "presença"(41), isto é, o quão realisticamente presente sentese o observador num ambiente virtual, ao fazer uso de um HMD. Muitas destas explicações hipotetizadas têm sido estudadas, mas nenhuma delas tem fornecido uma explicação completa e satisfatória, se alguma existe, considerando as diferenças no desempenho no ambiente real e no ambiente virtual. Importante, porém, é notar que nenhum estudo investigou a percepção de distâncias exocêntricas, apenas distâncias egocêntricas, absolutas. Também, nenhum estudo analisou o efeito da idade sobre os julgamentos de distância em espaço virtual, especialmente comparando indicadores, distâncias egocêntricas e exocêntricas, e em ambientes visuais reais e virtuais. Todavia, parte desta lacuna está sendo preenchida pelo trabalho em andamento por Aznar-Casanova., o qual tem comparado estimativas de distância exocêntrica em três arranjos ambientais: no plano fronto-paralelo (tela do computador), espaço virtual e em campo aberto $^{(42)}$.

\section{Da mensuração de distância em ambiente pictórico}

A questão central que pode ser formulada é: É a percepção de distância em espaço fotográfico igual àquela em espaço real?. Resposta a esta questão pode ser benéfica tanto para o entendimento da própria percepção pictórica quanto para o nosso conhecimento dos processos perceptuais gerais no mundo natural ${ }^{(2,43-45)}$. Em termos de aplicação, há uma crescente necessidade de construir equipamentos capazes de criar 
estimulações visuais verídicas e pedagogicamente eficazes para treinamento de habilidades que dependem fortemente da orientação visual. Em todas estas simulações de treinamento visual, desde a educação do motorista ao treino de pilotos de aviões, uma representação apropriada de distância é crítica. Por isso, é, certamente, importante examinar sistematicamente a natureza da translação do mundo natural no mundo pictórico-fotográfico.

No estudo de Kraft et al., foram examinados os efeitos de quatro lentes focais $(48,28,24 \mathrm{e} 17 \mathrm{~mm})$ sobre as estimativas de distâncias de objetos reais (por exemplo, árvores), configuradas em diapositivos coloridos de cenas em ambientes naturais ${ }^{(46)}$. Os observadores foram instruídos a se imaginarem na cena configurada e a julgarem quão distante eles estavam dos alvos indicados. Seus dados revelaram que a percepção de distância em profundidade foi sistematicamente transformada mudando o comprimento focal das lentes; de maneira que quanto menor o comprimento focal das lentes da câmera, maior a distância percebida de objetos na cena. Num outro estudo similar, foi examinada a percepção de distância de objetos representados fotograficamente alterando a área de visão fotográfica ${ }^{(47)}$. A área de visão foi manipulada fotografando cada cena com lentes de cinco comprimentos focais variando de: 135, 80, 48, 28 a $17 \mathrm{~mm}$. A percepção de distância em profundidade pictórica foi sistematicamente transformada alterando-se a área de visão fotográfica: quanto menor o comprimento focal da lente da câmera, mais ampla a área de visão, e maior a distância percebida. Interessante observar que modelando os dados por meio de funções de potência, a distância percebida em diapositivos fotográficos é governada como uma função negativamente acelerada da distância real. Além disso, a curvatura da função de distância, geralmente, aumenta quando o comprimento focal diminui e a área de visão fotográfica amplia-se. Todavia, a diminuição do expoente da função de potência pode ser explicada simplesmente pelo aumento na amplitude de distância fotográfica ${ }^{(13,48)}$. Isto é, quando o comprimento focal diminui, a fotografia parece representar uma amplitude maior de distâncias, e o expoente da função de potência diminui em resposta a este aumento da amplitude do estímulo.

Cutting ${ }^{(43)}$, discutindo estes dados obtidos em ambientes pictóricos e comparando-os com aqueles revisados por $\mathrm{Da}$ Silva ${ }^{(13)}$ e Wagner ${ }^{(49)}$ em ambientes reais, afirma que não é mera coincidência que os julgamentos de distância em fotografias e os julgamentos de distância no mundo real sejam tão similares. Também, ele entende que não é mera coincidência a similaridade encontrada entre os dados fotográficos e os dados do mundo real, no declínio dos expoentes encontrados truncando-se a distância próxima. Segundo Cutting a convergência entre estes dois conjuntos de dados não é certamente uma mera coincidência. Para ele, esta forte similaridade, indica uma identidade funcional entre o espaço fotográfico e o espaço do mundo real ${ }^{(43)}$.

Todavia, Watanabe tem publicado dados indicando que a profundidade percebida na fotografia não se assemelha à profundidade do espaço físico, bem como àquela do espaço visual, mas é mais similar à profundidade do espaço visual do que àquela do espaço físico ${ }^{(50)}$. Além disso, o espaço fotográfico tem uma propriedade anisotrópica tal como ocorre com espaço visual.

\section{Da superconstância de distância percebida}

As questões principais que tentaremos responder nessa seção são: Há uma tendência em direção à superconstância? O grau de superconstância depende exclusivamente da idade dos observadores, ou a amplitude de distância também a afeta?

De fato, como mencionamos alhures, usando a função de potência para a modelagem dos julgamentos de distância pode-se mostrar que há três possíveis relações funcionais entre distância percebida e distância física ou real. A distância percebida pode ser uma função desacelerada (subconstância), uma função linear (constância perfeita) ou uma função acelerada (superconstância) ${ }^{(13-14,17-19)}$. Todavia, estas relações funcionais têm sido influenciadas pela amplitude de distância, pelo tipo de indicador (método) de distância, pelo ambiente em que as distâncias são julgadas e pela natureza ou combinações de indícios presentes nestes ambientes.

A maioria destas relações funcionais é baseada sobre o desempenho de adultos. Ainda que poucos dados estejam disponíveis para a percepção de distância em crianças, eles sugerem que mudanças na acurácia da percepção de distância acompanham mudanças com a idade, seja nas próprias estimativas, seja na sua variabilidade. Mas, a direção e a taxa de mudança parecem também depender de vários fatores da situação experimental. Um dos mais relevantes é a amplitude de distância utilizada para julgamentos de distância egocêntrica. Tem sido conjecturado que, dentro do espaço pessoal e para pequenas distâncias dentro do espaço de ação ${ }^{(20)}$, ocorre invariavelmente, para adultos, uma superestimação (erros positivos) de distâncias percebidas, um fenômeno que tem sido rotulado de superconstância. Este fenômeno pode ser descritivamente analisado por meio do expoente (n) da função de potência, o qual também pode ser apropriadamente utilizado como um índice discriminativo da evolução perceptiva de distância, de subconstância para superconstância ${ }^{(51-52)}$. Podemos supor que, quanto mais jovem a criança tanto menor será o expoente que governa seus julgamentos de distância, e para crianças mais velhas, maior o expoente; para adolescentes e adultos, cujo comportamento perceptual caracteriza-se por uma tendência em direção a superconstância, esperar-se-iam expoentes maiores que a unidade. Assim, pois, a subconstância, que se supõe ser uma característica perceptual das crianças mais jovens, teria um expoente menor que 1,0 , valor que aumentaria à medida que a criança for se tornando mais velha. Além de ser afetada pela idade, esta tendência em direção a superconstância também é afetada pela amplitude de distância, de modo que quanto maior for a amplitude, menor será o expoente. Mas, a amplitude de distância não afeta igualmente os diferentes grupos etários. 
Os estudos de Harway ${ }^{(53)}$ e Collins ${ }^{(54)}$ investigaram percepção de distância em função da idade, mas eles não tiveram a intenção de comparar escalas de distância obtidas com crianças com aquela obtida para adultos. O único estudo que comparou grupos de diferentes idades foi aquele de Teghtsoonian e Beckwith ${ }^{(52)}$. Neste estudo, observadores entre oito e 18 anos de idade num ginásio de $17 \mathrm{~m}$ de comprimento fizeram estimativas diretas de magnitude para distâncias até $15,2 \mathrm{~m}$. Os resultados mostraram que tanto os observadores mais jovens quanto os adultos, todos julgaram distâncias igualmente. $\mathrm{O}$ expoente médio foi 0,93 ; isto é, a função foi levemente desacelerada.

Todavia, porque a magnitude dos principais indícios de distância é uma função negativamente acelerada da distância física, e como as crianças, diferentemente dos adultos, não têm tido experiência suficiente para desenvolver uma correção cognitiva de seus julgamentos de distância, diferentes expoentes para os vários grupos etários poderiam ter sido obtidos com distância maiores que aquelas usadas por Teghtsoonian e Beckwith. Na verdade, à luz dos argumentos apresentados por Wohlwill( ${ }^{(5)}$ e sistematicamente investigados por Gogel ${ }^{(56-57)}$, de que o grau de subconstância ou de superconstância depende não apenas da idade, mas também da amplitude de distância, podemos acreditar que para distância maiores (vista espaço) ${ }^{(20)}$ dispostas em ambiente real, as crianças mais velhas exibiriam subconstância: isto é, elas mostrariam expoentes menores que 1,0 .

Estas hipóteses foram investigadas por Da Silva ${ }^{(51)}$. Em seu estudo, cinco grupos de observadores cujas idades variaram de seis, oito, 10 e 12 anos de idade e adultos, estimaram num ambiente real (um grande campo aberto de $30 \times 300 \mathrm{~m}$ ) as distâncias egocêntricas de um, dois, quatro, oito, 16, 32, 64, 128,256 e $296 \mathrm{~m}$, englobando uma amplitude de 2,17 unidades logarítmicas. As distâncias foram demarcadas por triângulos de diferentes tamanhos. As distâncias foram estimadas pelo método de fracionamento (bipartição). Os expoentes médios foram: 0,55 para o grupo de seis anos, 0,59 para o grupo de oito e de 10 anos, 0,62 para o grupo de 12 anos e de 0,74 para o grupo de adultos. $\mathrm{O}$ expoente para o grupo de adultos difere de todos os demais grupos e aquele para o grupo de 12 anos difere do de seis anos de idade. Todas as demais comparações não foram significativas. Assim, embora os expoentes gradualmente aumentem com a idade, as diferenças entre os três grupos mais jovens não foram estatisticamente significativas. A diferença entre o grupo de adultos e os quatro grupos mais jovens, assim como a diferença entre o grupo de 12 anos e o grupo de seis anos, refletem uma mudança evolutiva. Quando todos os grupos são considerados, o expoente é menor que 1,0 para aproximadamente $98 \%$ dos observadores, e o expoente médio considerando todos os observadores é estatisticamente menor que o valor teórico de 1,0 o qual indica uma perfeita constância. Conseqüentemente, a distância percebida é uma função negativamente acelerada da distância real para todos os grupos etários, porém, os dados indicaram que as escalas de distância não são as mesmas para crianças como para adultos. Todavia, distância percebida em ambiente natural em grandes distâncias é caracterizada pela subconstância. Esta subconstância parece ser maior para os grupos de crianças mais jovens porque seus expoentes médios são menores que aqueles para adultos. Claramente, não há uma tendência evolutiva em direção a superconstância desde que as principais diferenças são entre os adultos e os quatro grupos de crianças mais jovens, e entre os grupos de 12 anos e o de seis anos de idade. Provavelmente, o expoente médio para adultos é maior que aqueles para crianças por causa de seu uso mais eficiente dos indícios cognitivos, não-perceptuais, de distância (por exemplo, tamanho familiar do experimentador, da textura do campo aberto, do tamanho relativo dos marcadores de distância) que inevitavelmente estão presentes em experimentos usualmente realizados em ambientes reais, naturais. Também, há subconstância para os adultos porque em grandes distâncias há poucos indícios de distância e correção cognitiva torna-se insuficiente para manter a constância de distância. Em outras palavras, indícios de distância que fortemente operam no espaço peripessoal e no espaço de ação, perdem sua robustez à medida que as distâncias físicas aumentam. Além disso, uma combinação de vários indícios presentes em ambientes reais, não é suficiente para manter a constância perfeita, mesmo para adultos, quando distâncias muito grandes estão envolvidas ${ }^{(20-21,56-57)}$.

Uma questão permanece, todavia, ainda sem resposta: Qual a relação psicofísica entre distância percebida e distância física em ambiente virtual para crianças de diferentes idades? Não há, até onde conhecemos, qualquer estudo publicado que investigou esta relação manipulando a idade dos observadores. Por isso, a resposta fica em aberto. Todavia, a partir de dados que compararam os julgamentos de adultos e de crianças em ambiente natural, e em adultos, comparam os julgamentos efetuados em ambiente real e em ambiente virtual, poderíamos fazer duas inferências: (1) haveria, para as crianças, uma forte compressão da distância quando comparada com aquela observada para adultos em ambiente virtual, e (2) esta compressão da distância egocêntrica estimada em ambiente virtual variará, provavelmente, em função da idade dos observadores, sendo maior para o grupo mais jovem.

\section{CONCLUSÕES}

O espaço visual é caracterizado por propriedades geométricas percebidas, principalmente distância egocêntrica e exocêntrica, além de tamanho, forma, orientação, etc. Intuitivamente, parece que estas propriedades são o resultado de uma transformação direta das características Euclidianas do espaço físico. ${ }^{(58)}$ Esta suposição, todavia, é inconsistente com uma variedade de intrigantes e, freqüentemente, sutis discrepâncias entre as conseqüências preditas de qualquer mapeamento direto do espaço físico e o que as pessoas realmente vêem.

De fato, considerando a distância percebida, um dos aspectos mais simples do espaço visual, mostramos que a dis- 
tância percebida (egocêntrica ou exocêntrica) não guarda uma simples relação com a sua contraparte física a partir do observador. Assim, quando os observadores são solicitados a fazer julgamentos em ambientes com poucos (condições reduzidas de indícios visuais) ou com indícios completos de distância (real ou física, virtual e pictórico-fotográfico, por exemplo), as distâncias indicadas diferem em vários modos das correspondentes distâncias físicas. Primeiro, objetos julgados em ambientes extremamente reduzidos em indícios visuais são tipicamente percebidos como estando numa distância de dois a quatro metros, um fenômeno denominado de tendência da distância específica ${ }^{(10,15-16,21)}$. Segundo, a distância percebida de um objeto a partir do observador parece ser, aproximadamente, igual à distância de objetos vizinhos na imagem retiniana, um fenômeno denominado de tendência da eqüidistância $^{(10,15-16,21)}$. Terceiro, quando apresentada no, ou próxima ao, nível do olho, a distância de um objeto relativamente próximo do observador tende a ser superestimada, ao passo que a distância de um objeto que está mais distante tende a ser subestimada $^{(13,20,32)}$. Quarto, a distância julgada de objetos dispostos no chão varia com o ângulo de declinação da linha de visão ${ }^{(26)}$; objetos que estão pelo menos alguns metros distantes parecem mais próximos do que realmente estão, e, progressivamente, mais elevados do que indicados pela sua posição física ${ }^{(59)}$. Quinto, sob condições em ambiente real, a distância percebida de objetos no chão é influenciada pela estrutura interveniente da superfície do terreno ${ }^{(59-60)}$. Sexto, julgamentos visualmente dirigidos (por exemplo, registro verbal ou atirar um alvo a uma distância previamente fixada) são substancialmente menos precisos do que os julgamentos feitos por ações visualmente dirigidas (por exemplo, caminhar com os olhos fechados a um alvo cuja distância está previamente fixada ou por triangulação) ${ }^{(1)}$. Sétimo, os julgamentos feitos em ambientes virtuais mostram uma maior compressão do que aqueles feitos em ambientes reais ${ }^{(61-66)}$. Oitavo, a compressão de distância egocêntrica em ambientes reais varia em função da idade do observador, sendo maior para grupos de observadores mais jovens, e, também, varia em função da amplitude de distância ${ }^{(51-52)}$. Nono, uma variedade de explicações tem sido proposta, mas há pouca ou nenhuma concordância sobre a base desta fenomenologia ${ }^{(8,43,62)}$. Finalmente, décimo, muitas pesquisas são ainda necessárias para que possamos enriquecer o nosso conhecimento e entendimento do sistema visual, especialmente como a distância é mapeada em distância percebida, seja ela egocêntrica ou exocêntrica. Para muitas aplicações práticas dos estudos envolvendo realidade virtual, a percepção espacial verídica é bastante crucial. Por conseqüência, um completo entendimento sobre os mecanismos determinando a percepção espacial em sistemas de ambiente virtual é necessário. Todavia, as pesquisas em percepção do espaço em ambientes virtuais estão apenas começando, e muito mais é necessário para que possamos pensar em aplicações. Estamos, certamente, otimistas e trabalhando para isso.

\section{AGRADECIMENTOS}

Este manuscrito é dedicado ao Doutor Harley E. A. Bicas, Professor Titular da Faculdade de Medicina de Ribeirão Preto, da Universidade de São Paulo, o qual, há mais de 40 anos, tem intensa e dinamicamente contribuído para o desenvolvimento e expansão das pesquisas nas áreas de percepção visual, psicofísica visual e visão, especialmente no Brasil e na América Latina.

\section{ABSTRACT}

The major aim of this overview was the visual perception of egocentric (distance from an observer to a target) and exocentric distance (distance between two targets). We considered different issues concerning the relationship between perceived distance and physical distance, giving special attention to the theories, to the cues regarding distance, how perceived distances are measured, and the types of visual environments where the measuring of distances occurred. We concluded that the perceived distance does not reflect a simple transformation of its physical counterpart; rather, the mapping between perceived distance and physical distance depends substantially on the type of visual environments where distances are measured, and, on the cue combination available in these environments.

Keywords: Depth perception; Space perception; Visual perception; Distance perception; Psychophysics, Optical illusions

\section{REFERÊNCIAS}

1. Loomis JM, Da Silva JA da, Fujita N, Fukusima SS. Visual space perception and visually directed action. J Exp Psychol Hum Percept Perform. 1992. 18(4):906-21

2. Gibson JJ. The ecological approach to visual perception: Hillsdate: Lawrence Erlbaum Associates; 1986

3. Gilinsky AS. Perceived size and distance in visual space. Psychol Rev. 1951; 58(6):460-82.

4. Indow T. The global structure of visual space. New Jersey: World Scientific; 2004. (Advanced Series on Mathematical Psychology, 1).

5. Goodale MA, Milner AD. Separate visual pathways for perception and action. Trends Neurosci. 1992;15(1):20-5.

6. Milner AD, Goodale MA. The visual brain in action. Oxford: Oxford University Press; 1995.

7. Michaels CF. Information, perception, and action: What should ecological psychologists learn from Milner and Goodale (1995)? Ecol Psychol. 2000; 12(3):241-58.

8. Sedgwick HA. Space perception. In: Boff KR, Kaufman L, Thomas JP, editors. Handbook of perception and human performance: sensory processes and perception. New York: Wiley; 1986. p.21.1-21.57.

9. Sedgwick HA. Visual space perception. In: Goldstein BE, editor. Handbook of perception. Oxford: Blackwell; 2001. p.129-67. (Handbooks in Experimental Psychology Series).

10. Gogel WC. The analysis of perceived space. In: Masin SC, editor. Foundations of perceptual theory. Amsterdam: Elsevier; 1993. p.113-82. (Advances in Psychology).

11. Loomis JM, Philbeck JW. The anisotropy of perceived 3-D shape invariant across scale?. Percept Psychophys. 1999;61(3):397-402.

12. Stevens SS. Psychophysics: Introduction to its perceptual, neural, and social prospects. 2000 ed. New York: John Willey and Sons; 1975. 
13. Da Silva JA. Scales for perceived egocentric distance in a large open field: comparison of three psychophysical methods. Am J Psychol. 1985;98(1):119-44.

14. Flückiger M. La perception d'objects lointains. In: Flückiger M, Klaue K, editors. La perception de l'environnement. Lausanne, Swtzerland: Delachaux et Niestlè; 1991. p.221-38.

15. Gogel WC, Da Silva JA. Familiar size and the theory of off-sized perceptions. Percept Psychophys. 1987;41(4):318-28.

16. Gogel WC, Da Silva JA. A two-process theory of the response to size and distance. Percept Psychophys. 1987;41(3):220-38.

17. Teghtsoonian M, Teghtsoonian R. Scaling apparent distance in natural indoor settings. Psychon Sci. 1969;16(6):281-3.

18. Teghtsoonian R, Teghtsoonian M. Scaling apparent distance in a natural outdoor setting. Psychon Sci. 1970;21(4):215-6.

19. Weist WM, Bell B. Stevens's exponent for psychophysical scaling of perceived, remembered, and inferred distance. Psychol Bull. 1985;98(3):457-70.

20. Cutting J, Vishton PE. Perceiving layout and knowing distances: the integration, relative potency, and contextual use of different information about depth. In: Epstein W, Rogers SP, editors. Perception of space and motion. San Diego CA: Academic Press; 1995. p.66-117.

21. Da Silva JA. Acerca da distinção entre fatores perceptivos e cognitivos na percepção de tamanho e distância. In: Biasoli-Alves ZMM, Da Silva JA, editores. Percepção: múltiplas visões. Ribeirão Preto: Legis Summa; 1990. p.53-72.

22. Beall AC, Loomis JM, Philbeck JW, Fikes TG. Absolute motion parallax weakly determines visual scale in real and virtual environments. In: The International Society for Optical Engineering: SPIE; Proceedings. 1995. p.288-97.

23. Gogel WC. Convergence as a cue to the perceived distance of objects in a binocular configuration. Rep US Army Med Res Lab. 1961;468:1-15.

24. Foley JM. Binocular distance perception. Psychol Rev. 1980;87(5):411-34.

25. Wu B, Ooi TL, He ZJ. Perceiving distance accurately by a directional process of integrating ground information. Nature. 2004;428(6978):73-7.

26. Ooi TL, Wu B, He ZJ. Distance determined by the angular declination below the horizon. Nature. 2001;414(6860):197-200. Comment in: Nature. 2001; 414(6860);155-6.

27. Predebon J. Relative distance judgments of familiar and unfamiliar objects viewed under representatively natural conditions. Percept Psychophys. 1990; 47(4):342-8

28. Predebon J. The familiar-size cue to distance and stereoscopic depth perception. Perception. 1993;22(8):985-95.

29. Matsushima EH, Olivera AP de, Ribeiro-Filho NP, Da Silva JA da. Visual angle as determinant factor for relative distance perception. Psicológica. 2005; 26(1):97-104.

30. Jacobs A, Shiffrar M. Walking perception by walking observers. J Exp Psychol Hum Percept Perform. 2005;31(1):157-69.

31. Fukusima SS, Loomis JM, Da Silva JA da. Visual perception of egocentric distance as assessed by triangulation. J Exp Psychol Hum Percept Perform. 1997;23(1):86-100.

32. Philbeck JW, Loomis JM. Comparison of two indicators of perceived egocentric distance under full-cue and reduced-cue conditions. J Exp Psychol Hum Percept Perform. 1997;23(1):72-85.

33. Thomson JA. Is continuous visual monitoring necessary in visually guided locomotion? J Exp Psychol Hum Percept Perform. 1983;9(3):427-43.

34. Rieser JJ, Ashmead DH, Talor CR, Youngquist GA. Visual perception and the guidance of locomotion without vision to previously seen targets. Perception. 1990;19(5):675-89.

35. Rieser JJ. Dynamic spatial orientation and the coupling of representation and action. In: Golledge RG, editor. Wayfinding behavior: cognitive mapping and other spatial processes. Baltimore: John Hopkins University Press; 1999. p.32-47.

36. Da Silva JA, Marques SS, Ruiz EM. Subject differences in exponents of the psychophysical power functions for inferred, remembered, and perceived area. Bull Psychon Soc. 1987;25:191-4.

37. Da Silva JA, Ruiz EM, Marques SL. Individual differences in magnitude estimates in inferred, remembered, and perceived geographical distance. Bull Psychon Soc. 1987;25:240-3.

38. Knapp JM, Loomis JM. Limited field of view of head-mounted displays is not the cause of distance underestimation in virtual environments. Presence: Teleoper Virtual Environ. 2004;41:25-38

39. Loomis JM, Blascovich JJ, Beall AC. Immersive virtual environment techno$\log$ as a basic research tool in psychology. Behav Res Methods Instrum Comput. 1999;31(4):557-64

40. Nakamizo S, Imamura M. Verification of Emmert's law in actual and virtual environments. J Physiol Anthropol Appl Human Sci. 2004;23(6):325-9.

41. Welch RB. How can we determine if the sense of presence affects task performance?. Presence: Teleoper Virtual Environ. 1999;8(5):574-7.

42. Aznar-Casanova JA. Distortion of visual space. In: 1 Iberian Congress on Perception; 2005 July 6-8; Barcelona, Spain.

43. Cutting J. Reconceiving perceptual space. In: Atherton M, Hecht H, Schwartz $\mathrm{R}$, editors. Perceiving pictures: an interdisciplinary approach to pictorial space. Cambridge, MA: MIT Press; 2004. p.215-38.

44. Gibson JJ. The information available in pictures. Leonardo. 1971;4:27-35.

45. Hagen MA. Picture perception: toward a theoretical model. Psychol Bull. 1974;81(8):471-97.

46. Kraft RN, Patterson JF, Mitchell NB. Distance perception in photographic displays of natural settings. Percept Mot Skills. 1986;62(1):179-86.

47. Kraft RN, Green JS. Distance perception as a function of photographic area of view. Percept Psychophys. 1989;45(5):459-66.

48. Teghtsoonian R. Range effects in psychophysical scaling and revision of Stevens' law. Am J Psychol.1973;86(1):3-27.

49. Wagner M. The metric of visual space. Percept Psychophys. 1985;38(6):483-95.

50. Watanabe T. [Anisotropy in depth perception of photograph]. Shinrigaku Kenkyu. 2004;75(1):24-32. Japanese.

51. Da Silva JA. Scales for measuring subjective distance in children and adults in a large open field. J Psychol. 1983;113(2d Half):221-30.

52. Teghtsoonian M, Beckwith JB. Children's size judgments when size and distance vary: is there a developmental trend to overconstancy? J Exp Child Psychol.1976;22(1):23-39.

53. Harway NI. Judgments of distance in children and adults. J Exp Psychol. 1963;65:385-90.

54. Collins JR. Distance perception as a function of age. Aust J Psychol. 1976; 28(2):109-13.

55. Wohlwill JF. The development of "overconstancy" in space perception. In: Lipsitt LP, Spiker CC, editors. Advances in child development and behavior. New York: Academic Press; 1963. p.265-312.

56. Gogel WC. Cognitive factors in spatial responses. Psychology. 1974;17:213-25.

57. Gogel WC. The metric of visual space. In: Epstein W, editor. Stability and constancy in visual perception: mechanisms and processes. New York: Wiley; 1977. p.129-81.

58. Hershenson M. Visual space perception. Massachusetts: MIT; 1999.

59. Sinai MJ, Ooi TL, He ZJ. Terrain influences the accurate judgement of distance. Nature. 1998;395(6701):497-500.

60. Meng JC, Sedgwick HA. Distance perception mediated through nested contact relations among surfaces. Percept Psychophys. 2001;63(1):1-15.

61. Creem-Regehr SH, Willemsen P, Gooch AA, Thompson WB. The influence of restricted viewing conditions on egocentric distance perception: implications for real and virtual indoor environments. Perception. 2005;34(2):191-204.

62. Loomis JM, Knapp JM. Visual perception of egocentric distance in real and virtual environments. In: Hettinger LJ, Haas MW, editors. Virtual and adaptive environments: applications, implications and human performance. New Jersey: LEA; 2003. p.21-46.

63. Sahm CS, Creem-Regehr SH, Thompson WB, Willemsen P. Throwing versus walking as indicators of distance perception in similar real and in absolute distance perception in real and virtual environments. ACM Trans Appl Percept. 2005;2(1):35-45.

64. Tcheang L, Gilson SJ, Glennerster A. Systematic distortions of perceptual stability investigated using immersive virtual reality. Vision Res. 2005; 45(16):2177-89.

65. Thompson WB, Willemsen P, Gooch AA, Creem-Regehr SH, Loomis JM, Beall AC. Does the quality of the computer graphics matter when judging distance in visually immersive environments? Presence Teleoper Virtual Environ. 2004;13:560-71.

66. Witmer BG, Sadowski WJJ. Nonvisually guided locomotion to a previously viewed target in real and virtual environments. Hum Factors. 1998;40(3):478-88. 